Comment. Math. Helv. 73 (1998) 480-498

(C) 1998 Birkhäuser Verlag, Basel

0010-2571/98/030480-19\$1.50+0.20/0

Commentarii Mathematici Helvetici

\title{
Deforming abelian SU(2)-representations of knot groups
}

Michael Heusener and Jochen Kroll*

\begin{abstract}
The aim of this paper is to generalize a theorem of C. D. Frohman and E. P. Klassen ([FK91]) concerning deformations of abelian SU(2)-representations of knot groups into nonabelian representations. The proof of our main theorem makes use of a generalization of a result of X.-S. Lin ([Lin92]) which should be interesting in itself.
\end{abstract}

Mathematics Subject Classification (1991). 57M25, 57M05.

Keywords. Knot groups, group representations, SU(2).

\section{Introduction}

The aim of this paper is to study the following question: when is an abelian representation of a knot group in $\mathrm{SU}(2)$ a limit point of non-abelian representations?

Let $k \subset S^{3}$ be a tame knot and let $G:=\pi_{1}\left(S^{3} \backslash k\right)$ be its group. A homomorphism $\rho: G \rightarrow \mathrm{SU}(2)$ is called abelian if and only if its image is abelian. The space of abelian conjugacy classes of representations is parameterized by the closed interval $[0, \pi]$. More precisely, let $m \in G$ be a meridian and let $\alpha \in[0, \pi]$ be given. We define an abelian representation $\rho_{\alpha}: G \rightarrow \mathrm{SU}(2)$ by $\rho_{\alpha}(m)=\mathbf{e}^{\mathbf{i} \alpha}$, where

$$
\mathbf{e}^{i \alpha}:=\left(\begin{array}{cc}
e^{\mathbf{i} \alpha} & 0 \\
0 & e^{-\mathbf{i} \alpha}
\end{array}\right)
$$

We denote the Alexander polynomial of $k$ by $\Delta_{k}$. If $\rho_{\alpha}$ is a limit of non-abelian representations then $\Delta_{k}\left(e^{2 \mathbf{i} \alpha}\right)=0$ (see theorem 2.1). It is conjectured that this condition is also sufficient.

C. Frohman and E. Klassen (see [FK91]) proved the conjecture under the assumption that $e^{2 \mathbf{i} \alpha}$ is a simple root of $\Delta_{k}$.

The aim of this paper is to prove the following theorem:

* The first author was partially supported by a TMR Marie Curie fellowship of the European Commission 
Theorem 1.1. Let $k \subset S^{3}$ be a knot and let $\alpha \in[0, \pi]$ be such that $\Delta_{k}\left(e^{2 \mathbf{i} \alpha}\right)=0$. If the signature function $\sigma_{k}: S^{1} \rightarrow \mathbb{Z}$ changes its value at $e^{2 \mathbf{i} \alpha}$ then the abelian representation $\rho_{\alpha}$ is an endpoint of an arc of non-abelian representations $G \rightarrow$ $\mathrm{SU}(2)$.

The signature function changes its value at $\omega \in S^{1}$ if $\omega$ is a root of $\Delta_{k}$ of odd multiplicity (see section 2). Therefore, theorem 1.1 generalizes theorem 1.1 in [FK91]. However, there are non-abelian deformations of abelian representations which are not detected by theorem 1.1 . The 2 -bridge knot $\mathfrak{b}(49,17)$ has an abelian representation which is the limit of non-abelian representations but the signature function does not change its value at the corresponding zero of the Alexander polynomial (see [Bur90]).

The proof of theorem 1.1 makes use of a generalization of a result of X.-S. Lin (see [Lin92]): let $G$ be a knot group and let $m \in G$ be a meridian. A representation $\rho: G \rightarrow \mathrm{SU}(2)$ is called trace-free if $\operatorname{tr} \rho(m)=0$. In [Lin92] Lin defined an intersection number of the representation spaces corresponding to a braid representative of the knot. This number turns out to be a knot invariant denoted by $h(k)$. Roughly speaking, $h(k)$ is the number of conjugacy classes of non-abelian tracefree representations $G \rightarrow \mathrm{SU}(2)$ counted with sign. Moreover, Lin established the relation

$$
h(k)=\frac{1}{2} \sigma(k) .
$$

It was remarked by D. Ruberman that the construction can be generalized to representations of knot groups with the trace of the meridians fixed. In section 4 we shall establish this generalization. More precisely, for a given $\alpha \in(0, \pi)$, we will define an integer invariant $h^{\alpha}(k)$. This invariant counts the conjugacy classes of non-abelian representations $G \rightarrow \mathrm{SU}(2)$, such that $\operatorname{tr} \rho(m)=2 \cos \alpha$ (note that $\left.h(k)=h^{\pi / 2}(k)\right)$. Since the definition of $h^{\alpha}(k)$ is straightforward we will only explain the set-up. However, the significant part is the computation of $h^{\alpha}(k)$ for $\alpha \neq \pi / 2$. We shall present all the details needed to prove the following theorem.

Theorem 1.2. Let $k$ be a knot such that $\Delta_{k}\left(e^{2 \mathbf{i} \alpha}\right) \neq 0$. Then

$$
h^{\alpha}(k)=\frac{1}{2} \sigma_{k}\left(e^{2 \mathbf{i} \alpha}\right) .
$$

The proof of theorem 1.1 together with theorem 1.2 implies the following:

Corollary 1.3. Let $k \subset S^{3}$ be a knot. If there is an $\alpha \in[0, \pi]$ such that $\Delta_{k}\left(e^{2 \mathbf{i} \alpha}\right), \sigma_{k}\left(e^{2 \mathbf{i} \alpha}\right) \neq 0$ then there exists an arc of irreducible $\mathrm{SU}(2)$ representations $G \rightarrow \mathrm{SU}(2)$.

This paper is organized as follows: In section 2 the basic notation and facts are presented. The proof of theorem 1.1 and the definition of $h^{\alpha}(k)$ are contained in section 3. The last section includes the computation of the invariant $h^{\alpha}(k)$. 
Remark 1.4. Theorem 1.1 was proved independently by C. Herald using gauge theory (see [Her97]).

\section{Notations and facts}

In this section we present the notation and facts which are needed in the sequel.

\subsection{The signature function}

Let $k \subset S^{3}$ be a tame knot and let $F$ be a Seifert surface for $k$. Since $F$ is oriented we have a normal direction and we can push cycles on $F$ along this normal direction into the complement of $F$. This defines a homomorphism $H_{1}(F) \rightarrow H_{1}\left(S^{3} \backslash F\right)$, $x \mapsto x^{+}$(here $H_{*}(X):=H_{*}(X, \mathbb{Z})$ ). The Seifert pairing $H_{1}(F) \otimes H_{1}(F) \rightarrow \mathbb{Z}$ is given by $x \otimes y \mapsto l k\left(x, y^{+}\right)$where $l k$ is the linking number in $S^{3}$. By fixing a basis $\left\{a_{i} \mid 1 \leq i \leq 2 g\right\}$ of $H_{1}(F)$ the pairing is described by a $2 g \times 2 g$ matrix $V$ over $\mathbb{Z}$ ( $g$ denotes the genus of $F$ ). We call $V$ a Seifert matrix for $k$. The antisymmetric matrix $V-V^{T}\left(V^{T}\right.$ is the transposed matrix of $\left.V\right)$ is the intersection matrix of the basis $\left\{a_{i}\right\}$ in $H_{1}(F)$ (see [BZ85] for details).

The normalized Alexander polynomial for $k$ is given by $\Delta_{k}:=\operatorname{det}\left(t^{1 / 2} V-\right.$ $\left.t^{-1 / 2} V^{T}\right)$. Here, normalized stands for $\Delta_{k}(t)=\Delta_{k}\left(t^{-1}\right)$ and $\Delta_{k}(1)=1$.

Let $\omega \neq 1$ be a complex number. We now consider the hermitian matrix $H(\omega):=(1-\omega) V+(1-\bar{\omega}) V^{T}$. The $\omega$-signature $\sigma_{k}(\omega)$ of $k$ is defined to be the signature of $H(\omega)$ i.e $\sigma_{k}(\omega)=\operatorname{sig}(H(\omega))$. If $\omega \in S^{1} \backslash\{1\}$ we have

$$
\begin{aligned}
H(\omega) & =(1-\omega) V+(1-\bar{\omega}) V^{T} \\
& =\left(\omega^{-1 / 2}-\omega^{1 / 2}\right)\left(\omega^{1 / 2} V-\omega^{-1 / 2} V^{T}\right) .
\end{aligned}
$$

The Levine-Tristram signature function is the map $\sigma_{k}: S^{1} \rightarrow \mathbb{Z}$ given by $\sigma_{k}: \omega \mapsto \sigma_{k}(\omega)$ if $\omega \neq 1$ and $\sigma_{k}: 1 \mapsto 0$. Let $Z_{k}:=\left\{\omega \in S^{1} \mid \Delta_{k}(\omega)=0\right\}$ be the set of zeros of $\Delta_{k}$ on the unit circle. It follows from equation (1) that the signature function is constant on the components of $S^{1} \backslash Z_{k}$. For a given $e^{2 \mathbf{i} \beta} \in Z_{k}$ we use the expression " $\sigma_{k}$ change its value at $e^{2 \mathbf{i} \beta}$ " or " $\sigma_{k}$ jumps at $e^{2 \mathbf{i} \beta}$ " if

$$
\lim _{t \downarrow \beta} \sigma_{k}\left(e^{2 \mathbf{i} t}\right) \neq \lim _{t \uparrow \beta} \sigma_{k}\left(e^{2 \mathbf{i} t}\right) .
$$

Moreover, it can be seen that $\sigma_{k}(\omega)=0$ if $\omega$ lies in a small neighborhood of 1 (for details see [Gor77] and [Kau87]).

\subsection{Representation spaces}

Let $G$ be a finitely generated group. The space of all representations of $G$ in $\mathrm{SU}(2)$ is denoted by $R(G):=\operatorname{Hom}(G, \mathrm{SU}(2))$. Note that $R(G)$ is a topological 
space via the compact open topology where $G$ carries the discrete and SU(2) the usual topology. A representation $\rho \in R(G)$ is called abelian (resp. central), (resp. trivial) if and only if its image is an abelian (resp. central), (resp. trivial) subgroup of $\mathrm{SU}(2)$. Note that $\rho \in R(G)$ is abelian if and only if it is reducible. The set of abelian representations is denoted by $S(G)$ and the set of central representations by $C(G)$. Two representations $\rho, \varrho \in R(G)$ are said to be conjugate $(\rho \sim \varrho)$ if and only if they differ by an inner automorphism of $\mathrm{SU}(2)$. The group $\mathrm{SO}(3)=\mathrm{SU}(2) /\{ \pm 1\}$ acts on $R(G)$ via conjugation. Two representations are in the same $\mathrm{SO}(3)$-orbit if and only if they are conjugate. Let $\widetilde{R}(G):=R(G) \backslash S(G)$ be the set of non-abelian representations. The space of (non-abelian) conjugacy classes of representations from $G$ into $\mathrm{SU}(2)$ is denoted by $\mathfrak{R}(G)(\widehat{R}(G))$ i.e.

$$
\mathfrak{R}(G):=R(G) / \mathrm{SO}(3) \quad \text { and } \quad \widehat{R}(G):=\widetilde{R}(G) / \mathrm{SO}(3) .
$$

We can think of the map $\widetilde{R}(G) \rightarrow \widehat{R}(G)$ as a principal $\mathrm{SO}(3)$-bundle (see [GM92, 3.A] for details). The spaces $\widehat{R}(G)$ and $\mathfrak{R}(G)$ are semi-algebraic sets. Here a subset of $\mathbb{R}^{n}$ is called semi-algebraic if it is a finite union of finite intersections of sets defined by a polynomial equation or inequality (see [Heu97] for details).

If $k \subset S^{3}$ is a knot then let $R(k)$ be short for $R\left(\pi_{1}\left(S^{3} \backslash k\right)\right)$. As we need the following theorem several times in the sequel we state it here.

Theorem 2.1. Let $k \subset S^{3}$ be a knot and let $\rho_{\alpha} \in S(k)$ be given. If $\Delta_{k}\left(e^{2 \mathbf{i} \alpha}\right) \neq 0$, then a sufficiently small neighborhood of $\rho_{\alpha}$ consists entirely of points of $S(k)$.

Proof. See [Kla91, Theorem 19].

\subsection{Quaternions}

During this paper it is sometimes more convenient to work with the quaternions (which we denote by $\mathbb{H}$ ). We identify $\mathrm{SU}(2)$ with the unit quaternions $\operatorname{Sp}(1) \subset \mathbb{H}$, the isomorphism is given by

$$
\left(\begin{array}{cc}
a & b \\
-\bar{b} & \bar{a}
\end{array}\right) \mapsto a+b \mathbf{j}
$$

The Lie algebra of $\operatorname{Sp}(1)$ is the set $\mathbb{E}$ of pure quaternions and $\operatorname{Sp}(1)$ acts via Ad on $\mathbb{E}$ i.e. $\operatorname{Ad}(q) X=q X q^{-1}$ for $q \in \operatorname{Sp}(1)$ and $X \in \mathbb{E}$. The intersection $\mathbb{E} \cap \operatorname{Sp}(1)$ - the set of pure unit quaternions - which is homeomorphic to the 2 sphere will be denoted by $S^{2}$. More general we consider the argument function arg: $\mathrm{SU}(2) \rightarrow[0, \pi]$ given by $\arg (A)=\arccos (\operatorname{tr}(A) / 2)$. For $\alpha \in(0, \pi)$ we have $\Sigma_{\alpha}:=\arg ^{-1}(\alpha)$ is a 2 -sphere and $S^{2}=\Sigma_{\pi / 2}$.

Given two elements $X, Y \in \mathbb{E}$, there is a product formula: $X \cdot Y=-\langle X, Y\rangle+$ $X \times Y$ where $\langle X, Y\rangle$ denotes the scalar product of $X$ and $Y$ and $X \times Y$ their vector 
product in $\mathbb{E}$. Note that $\operatorname{Ad}(q)$ preserves the scalar product. For $\mathbf{e}^{\mathbf{i} \alpha} \in \mathrm{SU}(2)$ we identify the tangent space $T_{\mathbf{e}} \alpha\left(\Sigma_{\alpha}\right)=\operatorname{span}(\mathbf{j}, \mathbf{k})$ with $\mathbb{C}$ via the multiplication by $-\mathbf{j}$. Under this identification the action of $\operatorname{Ad}\left(\mathbf{e}^{\mathbf{i} \alpha}\right)$ transforms into rotation about the angle $2 \alpha$ i.e multiplication by $e^{2 \mathbf{i} \alpha}$.

For each quaternion $q \in \operatorname{Sp}(1)$ there is an angle $\alpha, 0 \leq \alpha \leq \pi$, and $Q \in S^{2}$ such that $q=\cos \alpha+\sin \alpha Q$. The pair $(\alpha, Q)$ is unique if and only if $q \neq \pm 1$. Let $(\alpha, Q):=\cos \alpha+\sin \alpha Q$ for short.

\subsection{Burau-matrix}

Let $\mathfrak{B}_{n}$ be the braid group of rank $n$ with the standard generators $\sigma_{1}, \ldots, \sigma_{n-1}$. For a given $\sigma \in \mathfrak{B}_{n}$ we denote by $\Phi^{\sigma}:=\left(\Phi_{i, j}^{\sigma}\right)_{1 \leq i, j \leq n} \in \mathrm{GL}(n, \mathbb{Z}(t))$ its Buraumatrix and by $\phi^{\sigma} \in \mathrm{GL}(n-1, \mathbb{Z}(t))$ its reduced Burau-matrix (see [BZ85]). We write $\Phi^{\sigma}$ in the form

$$
\Phi^{\sigma}=\left(\begin{array}{ll}
\mathbf{A} & \mathbf{B} \\
\mathbf{C} & \mathbf{D}
\end{array}\right)
$$

where $\mathbf{D}=\mathbf{D}(t)$ is a $(n-2) \times(n-2)$ matrix. The matrix $C^{\sigma}(t):=\left(\Phi^{\sigma}-\mathbf{E}\right)$ is a Jacobian for the closed braid $\sigma^{\wedge}$ where $\mathbf{E}$ denotes the identity matrix (see [BZ85]). Let $c_{i, j}^{\sigma}(t)$ be the determinant of the matrix which is obtained from $C^{\sigma}(t)$ by omitting its $i$-th row and its $j$-th column. It is convenient to state the following lemma which will be used in the sequel.

Lemma 2.2. Let the $c_{i, j}^{\sigma}:=c_{i, j}^{\sigma}(t)$ be defined as above. Then

$$
\begin{aligned}
& \text { 1. } c_{l, m}^{\sigma}=(-1)^{m+m^{\prime}+l+l^{\prime}} t^{l-l^{\prime}} c_{l^{\prime}, m^{\prime}}^{\sigma} \\
& \text { 2. } \operatorname{det}\left(\mathbf{E}-\phi^{\sigma}(t)\right)=\frac{t^{n}-1}{t-1} c_{1, n}^{\sigma} \\
& \text { 3. } c_{1,1}^{\sigma_{1}^{2} \sigma}=t^{2} c_{1,1}^{\sigma}+(t-1) \operatorname{det}(\mathbf{D}(t)-\mathbf{E}) .
\end{aligned}
$$

Proof. The lemma is proved using the identities

$$
\sum_{j=1}^{n} \Phi_{i, j}^{\sigma}=1 \text { and } \sum_{i=1}^{n} t^{i-1} \Phi_{i, j}^{\sigma}=t^{j-1}
$$

(see [BZ85] for details).

\section{Proof of the main Theorem}

Let $\sigma \in \mathfrak{B}_{n}$ be given and denote by $\sigma^{\wedge}$ the closed $n$-braid defined by $\sigma$. Let $F_{n}$ be a free group with basis $\mathrm{S}=\left\{s_{1}, \ldots, s_{n}\right\}$. The braid $\sigma$ induces a braid automorphism (still denoted by $\sigma) \sigma: F_{n} \rightarrow F_{n}$. It follows that $\sigma$ induces a diffeomorphism (still denoted by $\sigma)$ of $\mathrm{SU}(2)^{n}$ i.e.

$$
\sigma\left(A_{1}, \ldots, A_{n}\right)=:\left(\sigma\left(A_{1}\right), \ldots, \sigma\left(A_{n}\right)\right) .
$$


Example 3.1. Let $\sigma=\sigma_{1}^{-2} \in \mathfrak{B}_{2}$. The $\sigma\left(A_{1}, A_{2}\right)=:\left(\sigma\left(A_{1}\right), \sigma\left(A_{2}\right)\right)$ where $\sigma\left(A_{1}\right)=A_{2}^{-1} A_{1} A_{2}$ and $\sigma\left(A_{2}\right)=A_{2}^{-1} A_{1}^{-1} A_{2} A_{1} A_{2}$.

Note that the equation $\prod_{i=1}^{n} A_{i}=\prod_{i=1}^{n} \sigma\left(A_{i}\right)$ always holds.

It was observed by Lin that the fixed point set of $\sigma: \mathrm{SU}(2)^{n} \rightarrow \mathrm{SU}(2)^{n}$ can be identified with $R\left(\sigma^{\wedge}\right)\left[\operatorname{Lin} 92\right.$, Lemma 1.2]. Let $\left(A_{1}, \ldots, A_{n}\right) \in \operatorname{Fix}(\sigma)$ be given. It follows that $\operatorname{tr} A_{i}=\operatorname{tr} A_{j}$ if $\sigma^{\wedge}$ is a knot. Therefore we are interested in the following space:

$$
R_{n}:=\left\{\left(A_{1}, \ldots, A_{n}\right) \in \mathrm{SU}(2)^{n} \mid \operatorname{tr}\left(A_{i}\right)=\operatorname{tr}\left(A_{j}\right), 1 \leq i, j \leq n\right\} \backslash\{ \pm(\mathbf{E}, \ldots, \mathbf{E})\} .
$$

Since $\sigma\left(R_{n}\right)=R_{n}$ we obtain a diffeomorphism $\sigma: R_{n} \rightarrow R_{n}$. Its fixed point set can be identified with $R\left(\sigma^{\wedge}\right) \backslash C\left(\sigma^{\wedge}\right)$.

Remark 3.2. A central representation of a knot group can never be a limit of non-abelian representations since $\Delta_{k}(1)=1$ (see theorem 2.1).

For a given $\alpha \in(0, \pi)$ let

$$
R_{n}^{\alpha}:=\left\{\left(A_{1}, \ldots, A_{n}\right) \mid \operatorname{tr}\left(A_{i}\right)=2 \cos \alpha, 1 \leq i \leq n\right\} \subset R_{n} .
$$

The set $R_{n}^{\alpha} \subset R_{n}$ is a submanifold of codimension one. Let us consider the following subspaces of $R_{2 n}$ :

$$
\begin{aligned}
H_{n} & :=\left\{\left(A_{1}, \ldots, A_{n}, B_{1}, \ldots, B_{n}\right) \in R_{2 n} \mid A_{1} \cdots A_{n}=B_{1} \cdots B_{n}\right\}, \\
\Lambda_{n} & :=\left\{\left(A_{1}, \ldots, A_{n}, A_{1}, \ldots, A_{n}\right) \in R_{2 n}\right\}, \\
\Gamma_{\sigma} & :=\left\{\left(A_{1}, \ldots, A_{n}, \sigma\left(A_{1}\right), \ldots, \sigma\left(A_{n}\right)\right) \in R_{2 n}\right\}, \\
S_{n} & :=\left\{\left(A_{1}, \ldots, A_{2 n}\right) \in R_{2 n} \mid A_{i} A_{j}=A_{j} A_{i}, 1 \leq i, j \leq n\right\} .
\end{aligned}
$$

Moreover, for $\Theta \in\left\{H_{n}, \Gamma_{\sigma}, \Lambda_{n}, S_{n}\right\}$ let $\Theta^{\alpha}:=\Theta \cap\left(R_{n}^{\alpha} \times R_{n}^{\alpha}\right)$. It is obvious that the fixed point set of $\sigma: R_{n} \rightarrow R_{n}$ is given by $\Gamma_{\sigma} \cap \Lambda_{n} \subset H_{n}$.

The sets $S_{n} \subset R_{2 n}$ and $S_{n}^{\alpha} \subset R_{n}^{\alpha} \times R_{n}^{\alpha}$ are the subsets corresponding to the abelian representations. Each element of $S_{n}^{\alpha}$ is conjugate to an element of the form $\left(\mathbf{e}^{\epsilon_{1} \mathbf{i} \alpha}, \ldots, \mathbf{e}^{\epsilon_{2 n} \mathbf{i} \alpha}\right)$ where $\epsilon_{i} \in\{ \pm 1\}$.

Lemma 3.3. Let $n \in \mathbb{Z}, n \geq 2$ be given. The set $H_{n} \backslash S_{n}$ is a smooth manifold of dimension $4 n-2$ and $H_{n}^{\alpha} \backslash S_{n}^{\alpha}$ is a smooth manifold of dimension $4 n-3$. Moreover, $H_{n}^{\alpha} \subset H_{n}$ is a submanifold of codimension one.

Proof. Let $f_{n}: R_{2 n} \rightarrow \mathrm{SU}(2)$ be given by

$$
f_{n}:\left(A_{1}, \ldots, A_{n}, B_{1}, \ldots, B_{n}\right) \mapsto A_{1} \cdots A_{n} B_{n}^{-1} \cdots B_{1}^{-1}
$$

and denote by $f_{n}^{\alpha}$ its restriction to $R_{n}^{\alpha} \times R_{n}^{\alpha}$. Note that $R_{n}^{\alpha} \times R_{n}^{\alpha} \subset R_{2 n}$ is a submanifold of codimension one. 
For a given point $(\mathbf{A}, \mathbf{B}) \in H_{n}^{\alpha} \backslash S_{n}^{\alpha}$ the derivative $D_{(\mathbf{A}, \mathbf{B})} f_{n}^{\alpha}$ is surjective. This can be seen by a direct calculation (see [Lin92] or [Heu97]). The conclusion of the lemma follows from this fact.

By fixing an orientation of SU(2) we orient the 2-spheres $\Sigma_{\alpha}=\arg ^{-1}(\alpha)$ in the following way: the normal bundle $\nu_{\alpha}$ of $\Sigma_{\alpha} \subset \mathrm{SU}(2)$ is oriented by pulling back the orientation $-d x$ of $[0, \pi]$. We choose the orientation of $\Sigma_{\alpha}$ such that the orientations of the short exact sequence

$$
0 \rightarrow T \Sigma_{\alpha} \rightarrow T_{\Sigma_{\alpha}} \mathrm{SU}(2) \rightarrow \nu_{\alpha} \rightarrow 0
$$

fit together. The space $R_{n}^{\alpha} \cong \Sigma_{\alpha}^{n}$ is then oriented via the product orientation. Moreover, the orientation of $R_{n} \cong(0, \pi) \times\left(S^{2}\right)^{n}$ is obtained from the orientations of $R_{n}^{\alpha}$ and $[0, \pi]$. The manifolds $\Lambda_{n}, \Gamma_{\sigma} \cong R_{n}$ and $\Lambda_{n}^{\alpha}, \Gamma_{\sigma}^{\alpha} \cong R_{n}^{\alpha}$ are also oriented. By lemma 3.3 we can pull back the orientation of $\mathrm{SU}(2)$ to obtain an orientation of the normal bundle of $f_{n}^{-1}(\mathbf{E}) \backslash S_{n}$ (resp. $\left.\left(f_{n}^{\alpha}\right)^{-1}(\mathbf{E}) \backslash S_{n}^{\alpha}\right)$. This enables us to orient the manifolds $H_{n} \backslash S_{n}$ and $H_{n}^{\alpha} \backslash S_{n}^{\alpha}$. Now $\mathrm{SO}(3)$ acts fixed point free via conjugation on the oriented manifolds $\Theta \backslash S_{n}$ (resp. $\Theta^{\alpha} \backslash S_{n}^{\alpha}$ ) where $\Theta \in\left\{H_{n}, \Lambda_{n}, \Gamma_{\sigma}\right\}$. The action of $\mathrm{SO}(3)$ is orientation preserving ( $\mathrm{SO}(3)$ is connected) and we obtain the following oriented manifolds:

$$
\widehat{\Theta}:=\left(\Theta \backslash S_{n}\right) / \mathrm{SO}(3) \text { and } \widehat{\Theta}^{\alpha}:=\left(\Theta^{\alpha} \backslash S_{n}^{\alpha}\right) / \mathrm{SO}(3) .
$$

Remark 3.4. Since $\operatorname{dim} R_{2 n}=4 n+1$ and $\operatorname{dim} R_{n}^{\alpha}=2 n$ one has:

$$
\operatorname{dim} \widehat{\Lambda}_{n}=2 n-2, \quad \operatorname{dim} \widehat{\Gamma}_{\sigma}=2 n-2, \quad \operatorname{dim} \widehat{H}_{n}=4 n-5
$$

and

$$
\operatorname{dim} \widehat{\Lambda}_{n}^{\alpha}=2 n-3, \quad \operatorname{dim} \widehat{\Gamma}_{\sigma}^{\alpha}=2 n-3, \quad \operatorname{dim} \widehat{H}_{n}^{\alpha}=4 n-6
$$

The intersection number

$$
h^{\alpha}(\sigma):=\left\langle\widehat{\Lambda}_{n}^{\alpha}, \widehat{\Gamma}_{\sigma}^{\alpha}\right\rangle_{\widehat{H}_{n}^{\alpha}} \in \mathbb{Z}
$$

is well defined if the intersection $\widehat{\Lambda}_{n}^{\alpha} \cap \widehat{\Gamma}_{\sigma}^{\alpha} \subset \widehat{H}_{n}^{\alpha}$ is compact. It can be shown that $h^{\alpha}$ is indeed a knot invariant.

Proposition 3.5. Let $\sigma \in \mathfrak{B}_{n}$ and $\tau \in \mathfrak{B}_{m}$ be given such that $\sigma^{\wedge} \cong \tau^{\wedge}$ as knots.

If $\widehat{\Lambda}_{n}^{\alpha} \cap \widehat{\Gamma}_{\sigma}^{\alpha}$ is compact then $\widehat{\Lambda}_{m}^{\alpha} \cap \widehat{\Gamma}_{\tau}^{\alpha}$ is also compact and the intersection numbers $h^{\alpha}(\sigma)$ and $h^{\alpha}(\tau)$ are equal. 
Proof. Analogous to Lin's proof (see [Lin92, Theorem 18]).

The following lemma gives a criteria for the compactness of the intersection $\widehat{\Lambda}_{n}^{\alpha} \cap \widehat{\Gamma}_{\sigma}^{\alpha}$.

Lemma 3.6. Let $\sigma \in \mathfrak{B}_{n}$ be a braid such that $k:=\sigma^{\wedge}$ is a knot. If $\Delta_{k}\left(e^{2 \mathbf{i} \alpha}\right) \neq 0$ then

$$
\widehat{\Lambda}_{n}^{\alpha} \cap \widehat{\Gamma}_{\sigma}^{\alpha} \subset \widehat{H}_{n}^{\alpha}
$$

is compact.

Proof. The conclusion of the lemma follows direct from theorem 2.1.

Remark 3.7. It is possible for $\widehat{\Lambda}_{n}^{\alpha} \cap \widehat{\Gamma}_{\sigma}^{\alpha} \subset \widehat{H}_{n}^{\alpha}$ to be compact but $\Delta_{k}\left(e^{2 \mathbf{i} \alpha}\right)=0$. An example is given by the class of 2-bridge knots.

The space of non-abelian equivalence classes of representations $\widehat{R}\left(\sigma^{\wedge}\right)$ can be identified with the intersection $\widehat{\Lambda}_{n} \cap \widehat{\Gamma}_{\sigma} \subset \widehat{H}_{n}$. The intersection $\widehat{\Lambda}_{n} \cap \widehat{\Gamma}_{\sigma}$ is in general not compact. However, if the abelian representation $\rho_{\alpha}$ is not a limit of non-abelian representations the intersection $\widehat{\Lambda}_{n}^{\alpha} \cap \widehat{\Gamma}_{\sigma}^{\alpha} \subset H_{n}^{\alpha}$ is compact. Moreover, there exists an $\epsilon>0$ such that

$$
\left(\widehat{\Lambda}_{n} \cap \widehat{\Gamma}_{\sigma}\right) \cap \widehat{H}_{n}^{[\alpha-\epsilon, \alpha+\epsilon]}
$$

is compact where

$$
\widehat{H}_{n}^{\left[\alpha_{1}, \alpha_{2}\right]}:=\bigcup_{\alpha \in\left[\alpha_{1}, \alpha_{2}\right]} \widehat{H}_{n}^{\alpha} .
$$

This follows from the fact that $\mathfrak{R}(k)$ is a compact, semi-algebraic set.

Proposition 3.8. Let $\sigma \in \mathfrak{B}_{n}$ be given such that $\sigma^{\wedge}$ is a knot. Moreover, assume that $\rho_{\alpha}$ is not a limit of non-abelian representations.

Then there is an $\epsilon>0$ such that $h^{\alpha}(\sigma)=h^{\beta}(\sigma)$ for $|\alpha-\beta|<\epsilon$.

Proof. Choose an $\epsilon>0$ such that

$$
\left(\widehat{\Lambda}_{n} \cap \widehat{\Gamma}_{\sigma}\right) \cap \widehat{H}_{n}^{\alpha, \epsilon}
$$

is compact where $\widehat{H}_{n}^{\alpha, \epsilon}:=\widehat{H}_{n}^{[\alpha-\epsilon, \alpha+\epsilon]}$.

In general we have $\widehat{\Gamma}_{\sigma} \pitchfork \widehat{H}_{n}^{\beta}$ for all $\beta \in(0, \pi)$. Let $\widehat{\Gamma}_{\sigma}^{\alpha} \rightsquigarrow \widetilde{\Gamma}_{\sigma}^{\alpha}$ be an isotopy with compact support such that $\widetilde{\Gamma}_{\sigma}^{\alpha} \pitchfork \widehat{\Lambda}_{n}^{\alpha}$. Extend this deformation to an isotopy $\widehat{\Gamma}_{\sigma} \rightsquigarrow \widetilde{\Gamma}_{\sigma}$ with compact support such that $\widetilde{\Gamma}_{\sigma} \pitchfork_{\widehat{H}_{n}^{\alpha, \epsilon}} \widehat{\Lambda}_{n}$ and $\widetilde{\Gamma}_{\sigma} \pitchfork \widehat{H}_{n}^{\beta}$ for all $\beta \in(0, \pi)$. For a given $\beta \in(0, \pi)$ let $\widetilde{\Gamma}_{\sigma}^{\beta}:=\widetilde{\Gamma}_{\sigma} \cap \widehat{H}_{n}^{\beta}$. Note that $\widetilde{\Gamma}_{\sigma}^{\beta} \subset \widehat{H}_{n}^{\beta}$ is a $(2 n-3)$-dimensional manifold and $\widehat{\Lambda}_{n}^{\beta} \pitchfork \widetilde{\Gamma}_{\sigma}^{\beta}$ if $|\alpha-\beta|$ is sufficiently small. 
Now $\widehat{\Lambda}_{n} \cap \widetilde{\Gamma}_{\sigma}$ is an oriented one dimensional manifold in a neighborhood of $\widehat{H}_{n}^{\alpha}$. If $\beta$ is close to $\alpha$ then the one dimensional manifold $\widehat{\Lambda}_{n} \cap \widetilde{\Gamma}_{\sigma}$ yields a bordism in $\widehat{H}_{n}$ between $\widehat{\Lambda}_{n}^{\beta} \cap \widetilde{\Gamma}_{\sigma}^{\beta} \subset \widehat{H}_{n}^{\beta}$ and $\widehat{\Lambda}_{n}^{\alpha} \cap \widetilde{\Gamma}_{\sigma}^{\alpha} \subset \widehat{H}_{n}^{\alpha}$.

It follows that $h^{\alpha}(\sigma)=h^{\beta}(\sigma)$.

Proof of theorem 1.1. Assume that $\rho_{\alpha}$ is not the limit of non-abelian representations. By proposition 3.8 and by theorem 1.2 we obtain that the signature function does not jump at $e^{2 \mathbf{i} \alpha}$. This contradicts the hypothesis of the theorem 1.1.

A further consequence is the following:

Corollary 3.9. Let $k \subset S^{3}$ be a knot. If $\Delta_{k}\left(e^{2 \mathbf{i} \alpha}\right) \neq 0$ and if $\sigma_{k}\left(e^{2 \mathbf{i} \alpha}\right) \neq 0$ then there is a non-abelian representation $\rho_{0} \in \widehat{R}(k)$ such that $\operatorname{tr} \rho_{0}(m)=2 \cos \alpha$. Moreover, there is an arc $\rho_{t} \in \widehat{R}(k), t \in[-\epsilon, \epsilon]$, through $\rho_{0}$ such that $\operatorname{tr} \rho_{-\epsilon}(m)<$ $2 \cos \alpha$ and $\operatorname{tr} \rho_{\epsilon}(m)>2 \cos \alpha$.

Proof. The proof of the corollary can be derived from the proof of proposition 3.8 (see [Heu97, Theorem 5.10] for details).

Corollary 1.3 is an immediate consequence of corollary 3.9.

\section{The computation of $h^{\alpha}(k)$}

The aim of this section is to compute the invariant $h^{\alpha}(k)$. We will only treat the case $\alpha \neq \pi / 2$ but it is easy to see that the "exception" $\alpha=\pi / 2$ is included as a limit case (see remark 4.9).

The reference for this section is Lin's paper (see [Lin92]). We restrict ourself to the points which are different from the result proved by Lin. It should be remarked that the lemma 4.4 simplifies the proof. Lemma 4.4 applies also in the case $\alpha=\pi / 2$ and simplifies also Lin's proof.

The diploma thesis of the second author contains the main part of this section together with a more detailed discussion and many explicit examples (see [Kro96]).

The following lemma shows that the trace-free case is somehow special.

Lemma 4.1. Let $\alpha \in(0, \pi)$ be given. Then

(i): The space $\widehat{H}_{2}^{\pi / 2}$ is a 2-sphere with four cone points deleted.

(ii): For $\alpha \neq \pi / 2$ the space $\widehat{H}_{2}^{\alpha}$ is a 2-sphere with three points deleted.

Proof. Let $\left(\alpha, Q_{i}\right) \in \mathrm{Sp}(1), i=1, \ldots, 3$ be given. There exists an element $\left(\alpha, Q_{4}\right) \in$ $\operatorname{Sp}(1)$ such that $\left(\alpha, Q_{1}\right)\left(\alpha, Q_{2}\right)=\left(\alpha, Q_{3}\right)\left(\alpha, Q_{4}\right)$ if and only if

$$
\Re\left(\left(\alpha, Q_{3}\right)^{-1}\left(\alpha, Q_{1}\right)\left(\alpha Q_{2}\right)\right)=\cos \alpha .
$$


An easy calculation shows that this equation is equivalent to

$$
\gamma\left(1+\left\langle Q_{1}, Q_{2}\right\rangle\right)=\left\langle Q_{1} \times Q_{2}+\gamma\left(Q_{1}+Q_{2}\right), Q_{3}\right\rangle
$$

where $\gamma=\cot \alpha$. After conjugating we can assume that $Q_{2}=\mathbf{i}$ and $Q_{1}=\cos \theta_{1} \mathbf{i}+$ $\sin \theta_{1} \mathbf{j}$ where $0 \leq \theta_{1} \leq \pi$.

If $\alpha=\pi / 2$ the equation (2) reduces to $\left\langle\sin \theta_{1} \mathbf{k}, Q_{3}\right\rangle=0$. If $\sin \theta_{1} \neq 0$ this implies $Q_{3}=\cos \theta_{2} \mathbf{i}+\sin \theta_{2} \mathbf{j}$ where $0 \leq \theta_{2} \leq 2 \pi$. If $\sin \theta_{1}=0$ we obtain by conjugation $Q_{3}=\cos \theta_{2} \mathbf{i}+\sin \theta_{2} \mathbf{j}$ where $0 \leq \theta_{2} \leq \pi$. So, parameterized by $\theta_{1}$ and $\theta_{2}, \widehat{H}_{2}^{\pi / 2}$ is a pillowcase. The diagonal $\widehat{\Lambda}_{2}^{\alpha}$ is parameterized by $(\theta, \theta), 0<\theta<\pi$.

The conjugacy classes of abelian representations $S_{2}^{\pi / 2}$ are represented by the four points $A:=(\mathbf{i}, \mathbf{i}, \mathbf{i}, \mathbf{i}), A^{\prime}:=(\mathbf{i}, \mathbf{i},-\mathbf{i},-\mathbf{i}), B:=(\mathbf{i},-\mathbf{i},-\mathbf{i}, \mathbf{i})$ and $B^{\prime}:=(\mathbf{i},-\mathbf{i}, \mathbf{i},-\mathbf{i})$. Therefore, the four deleted points are given by the parameters $A=(0,0), A^{\prime}=$ $(0, \pi), B=(\pi, 0)$ and $B^{\prime}=(\pi, \pi)$.

If $\alpha \neq \pi / 2$ we set

$$
Q^{\prime}:=Q_{1} \times Q_{2}+\gamma\left(Q_{1}+Q_{2}\right)
$$

$Q\left(\alpha, \theta_{1}\right):=Q^{\prime} /\left\|Q^{\prime}\right\| \in S^{2}$ and $c\left(\alpha, \theta_{1}\right):=\frac{\gamma}{\left\|Q^{\prime}\right\|}\left(1+\left\langle Q_{1}, Q_{2}\right\rangle\right)$. More explicit we have

$$
Q\left(\alpha, \theta_{1}\right)=-\frac{\sin \left(\frac{\theta_{1}}{2}\right) \mathbf{k}}{\left(\sin ^{2}\left(\frac{\theta_{1}}{2}\right)+\gamma^{2}\right)^{1 / 2}}+\frac{\gamma\left(\cos \left(\frac{\theta_{1}}{2}\right) \mathbf{i}+\sin \left(\frac{\theta_{1}}{2}\right) \mathbf{j}\right)}{\left(\sin ^{2}\left(\frac{\theta_{1}}{2}\right)+\gamma^{2}\right)^{1 / 2}}
$$

and

$$
c\left(\alpha, \theta_{1}\right)=\frac{\gamma \cos \left(\frac{\theta_{1}}{2}\right)}{\left(\sin ^{2}\left(\frac{\theta_{1}}{2}\right)+\gamma^{2}\right)^{1 / 2}} .
$$

Note that $Q\left(\alpha, \theta_{1}\right)$ lies on the right bisector of the geodesic segment between $Q_{1}$ and $Q_{2}$. Moreover, $\left|c\left(\alpha, \theta_{1}\right)\right| \leq 1$ and

$$
\left|c\left(\alpha, \theta_{1}\right)\right|= \begin{cases}1 & \Leftrightarrow \theta_{1}=0 \\ 0 & \Leftrightarrow \theta_{1}=\pi\end{cases}
$$

It is obvious that the set

$$
E:=E\left(\alpha, \theta_{1}\right):=\left\{X \in \mathbb{E}^{3} \mid\left\langle Q\left(\alpha, \theta_{1}\right), X\right\rangle=c\left(\alpha, \theta_{1}\right)\right\}
$$

is a plane orthogonal to $Q\left(\alpha, \theta_{1}\right)$ and that $c\left(\alpha, \theta_{1}\right)$ is the oriented distance from the origin. We see that the points $Q_{3}$ which satisfies equation (2) are exactly those in the intersection $S^{2} \cap E$. The set $S^{2} \cap E$ is in general a small circle on the sphere $S^{2}$. This small circle degenerates to a point if and only if $\theta_{1}=0$ and to a great circle if and only if $\theta_{1}=\pi$ (see equation (3)). 
We set $\epsilon(\alpha):=\operatorname{sig} c\left(\alpha, \theta_{1}\right)=\operatorname{sig} \gamma \in\{ \pm 1\}$. For a given $Q_{3} \in S^{2} \cap E$ we can write

$$
Q_{3}=c\left(\alpha, \theta_{1}\right) \cdot Q\left(\alpha, \theta_{1}\right)+\cos \theta_{2} V_{1}+\sin \theta_{2} V_{2}
$$

where $V_{1}:=V_{1}\left(\alpha, \theta_{1}\right):=Q_{1}-c\left(\alpha, \theta_{1}\right) \cdot Q\left(\alpha, \theta_{1}\right)$ and $V_{2}=: V_{2}\left(\alpha, \theta_{1}\right):=\epsilon(\alpha) \cdot Q \times V_{1}$ (note that $Q_{1}, Q_{2} \in S^{2} \cap E$ ). So, $\theta_{2}$ is the oriented angle between $Q_{1}$ and $Q_{3}$ on $S^{2} \cap E$.

The parameterization degenerates when $Q_{1}=Q_{2}$ which is equivalent to $\theta_{1}=$ 0 . Furthermore, the representations corresponding to the parameters $\left(\pi, \theta_{2}\right)$ and $\left(\pi, 2 \pi-\theta_{2}\right)$ are equivalent (see figure 1$)$.

The conjugacy classes of abelian representations $S_{2}^{\alpha} \cap H_{2}^{\alpha}$ are represented by the three points $\left(\mathbf{e}^{\mathbf{i} \alpha}, \mathbf{e}^{\mathbf{i} \alpha}, \mathbf{e}^{\mathbf{i} \alpha}, \mathbf{e}^{\mathbf{i} \alpha}\right),\left(\mathbf{e}^{\mathbf{i} \alpha}, \mathbf{e}^{-\mathbf{i} \alpha}, \mathbf{e}^{-\mathbf{i} \alpha}, \mathbf{e}^{\mathbf{i} \alpha}\right)$ and $\left(\mathbf{e}^{\mathbf{i} \alpha}, \mathbf{e}^{-\mathbf{i} \alpha}, \mathbf{e}^{\mathbf{i} \alpha}, \mathbf{e}^{-\mathbf{i} \alpha}\right)$. Therefore, the three deleted points are given by the parameters $A=\left(0, \theta_{2}\right), B=$ $(\pi, \pi)$ and $B^{\prime}=(\pi, 0)$ where $0 \leq \theta_{2} \leq 2 \pi$. The diagonal $\widehat{\Lambda}_{2}^{\alpha}$ is parameterized by $(\theta, 0), 0<\theta<\pi$, connecting the points $A$ and $B^{\prime}$.

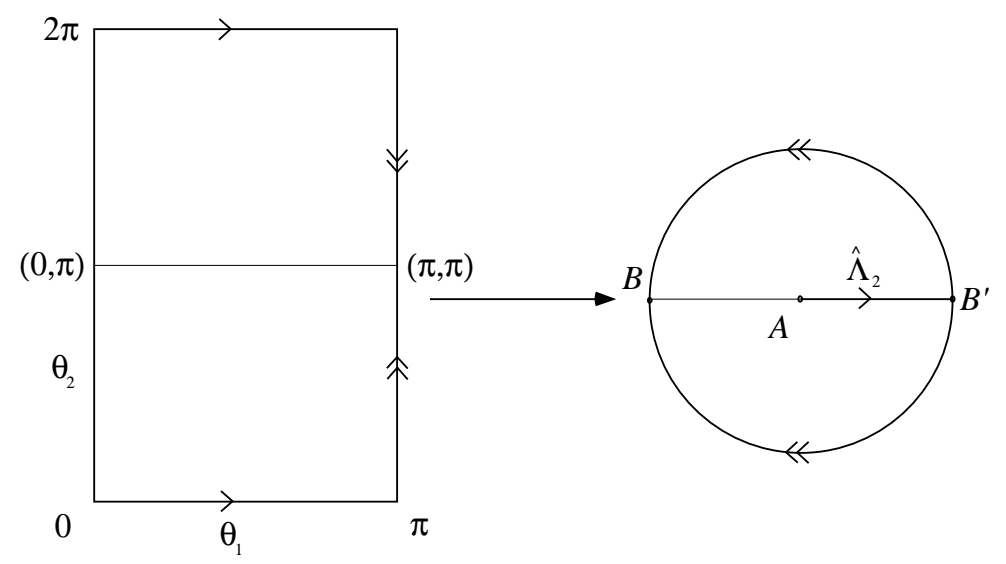

Figure 1.

Parameterization of $\widehat{H}_{2}^{\alpha}$

For the braid $\sigma_{1} \in \mathfrak{B}_{2}$ we obtain $h^{\alpha}\left(\sigma_{1}\right)=0$ because every point of $\Lambda_{2}^{\alpha} \cap \Gamma_{\sigma_{1}}^{\alpha}$ represents an abelian representation. Therefore the invariant vanishes for the trivial knot.

In order to determine the invariant for every knot we would like to study its behavior under crossing changes. Henceforth, let $\sigma \in \mathfrak{B}_{n}$ be a braid such that $k:=\sigma^{\wedge} \subset S^{3}$ is a knot. Then $k^{\prime}:=\left(\sigma_{1}^{2} \sigma\right)^{\wedge} \subset S^{3}$ is also a knot and we now study 
the difference

$$
\begin{aligned}
h^{\alpha}\left(\sigma_{1}^{2} \sigma\right)-h^{\alpha}(\sigma) & =\left\langle\widehat{\Lambda}_{n}^{\alpha}, \widehat{\Gamma}_{\sigma_{1}^{2} \sigma}^{\alpha}\right\rangle-\left\langle\widehat{\Lambda}_{n}^{\alpha}, \widehat{\Gamma}_{\sigma}^{\alpha}\right\rangle \\
& =\left\langle\widehat{\Gamma}_{\sigma_{1}^{-2}}^{\alpha}, \widehat{\Gamma}_{\sigma}^{\alpha}\right\rangle-\left\langle\widehat{\Lambda}_{n}^{\alpha}, \widehat{\Gamma}_{\sigma}^{\alpha}\right\rangle \\
& =\left\langle\widehat{\Gamma}_{\sigma_{1}^{-2}}^{\alpha}-\widehat{\Lambda}_{n}^{\alpha}, \widehat{\Gamma}_{\sigma}^{\alpha}\right\rangle
\end{aligned}
$$

Here we have chosen $\alpha \in(0, \pi), \alpha \neq \pi / 2$ such that $\Delta_{k}\left(e^{2 \mathbf{i} \alpha}\right) \neq 0$ and $\Delta_{k^{\prime}}\left(e^{2 \mathbf{i} \alpha}\right) \neq 0$.

Observe that the "difference cycle" $\left(\Gamma_{\sigma_{1}^{-2}}^{\alpha}-\Lambda_{n}^{\alpha}\right)$ is carried by

$$
V_{n}^{\alpha}:=\left\{\left(A_{1}, \ldots, A_{n}, B_{1}, \ldots, B_{n}\right) \in H_{n}^{\alpha} \mid A_{i}=B_{i}, i=3, \ldots, n\right\} .
$$

Then $\widehat{V}_{n}^{\alpha}:=\left(V_{n}^{\alpha} \backslash S_{n}^{\alpha}\right) / \mathrm{SO}(3)$ is a $(2 n-2)$-dimensional submanifold of $\widehat{H}_{n}^{\alpha}$. Note that there is a natural projection $p: V_{n}^{\alpha} \rightarrow H_{2}^{\alpha}$ given by

$$
p:\left(A_{1}, A_{2}, A_{3}, \ldots, A_{n}, B_{1}, B_{2}, A_{3}, \ldots, A_{n}\right) \mapsto\left(A_{1}, A_{2}, B_{1}, B_{2}\right) .
$$

To obtain a map between $\widehat{V}_{n}^{\alpha}$ and $\widehat{H}_{2}^{\alpha}$ we have to consider the set $W_{n}^{\alpha}:=p^{-1}\left(S_{2}^{\alpha} \cap\right.$ $\left.H_{2}^{\alpha}\right)$. It is easy to see that $W_{n}^{\alpha} \supset \Lambda_{n}^{\alpha} \cap \Gamma_{\sigma_{1}^{-2}}^{\alpha}$ is a $(2 n-2)$-dimensional manifold. Therefore $p$ induces a map $\widehat{p}: \widehat{V}_{n}^{\alpha} \backslash \widehat{W}_{n}^{\alpha} \rightarrow \widehat{H}_{2}^{\alpha}$ where $\operatorname{dim} \widehat{W}_{n}^{\alpha}=2 n-5$.

We apply the process used in [Lin92]: we perturb $\widehat{\Gamma}_{\sigma}^{\alpha}$ to $\widetilde{\Gamma}_{\sigma}^{\alpha}$ with compact support such that

$$
\left(\widehat{\Gamma}_{\sigma_{1}^{-2}}^{\alpha}-\widehat{\Lambda}_{n}^{\alpha}\right) \pitchfork \widetilde{\Gamma}_{\sigma}^{\alpha}
$$

which means precisely that $\widetilde{\Gamma}_{\sigma}^{\alpha} \pitchfork \widehat{\Gamma}_{\sigma_{1}^{-2}}^{\alpha}, \widehat{\Lambda}_{n}^{\alpha}$ and $\widetilde{\Gamma}_{\sigma}^{\alpha} \cap \widehat{W}_{n}^{\alpha}=\emptyset$. Moreover, we extend the isotopy so that $\widehat{V}_{n}^{\alpha} \pitchfork \widetilde{\Gamma}_{\sigma}^{\alpha}$. Thus $\widehat{V}_{n}^{\alpha} \cap \widetilde{\Gamma}_{\sigma}^{\alpha}$ is a 1-dimensional manifold.

We choose the orientations as in [Lin92]. It is sufficient to project the high dimensional manifolds via $\widehat{p}$ in order to study the difference $h^{\alpha}\left(\sigma_{1}^{2} \sigma\right)-h^{\alpha}(\sigma)$. More precisely, the following equation holds

$$
h^{\alpha}\left(\sigma_{1}^{2} \sigma\right)-h^{\alpha}(\sigma)=\left\langle\widehat{\Gamma}_{\sigma_{1}^{-2}}^{\alpha}-\widehat{\Lambda}_{2}^{\alpha}, \widehat{p}\left(\widetilde{\Gamma}_{\sigma}^{\alpha}\right)\right\rangle_{\widehat{H}_{2}^{\alpha}} .
$$

To study the intersection of $\left(\widehat{\Gamma}_{\sigma_{1}^{-2}}^{\alpha}-\widehat{\Lambda}_{2}^{\alpha}\right)$ with $\widehat{p}\left(\widetilde{\Gamma}_{\sigma}^{\alpha}\right)$ we have to understand the limiting behavior of the set $\widehat{p}\left(\widehat{\Gamma}_{\sigma}^{\alpha}\right)$ near the point $A$. In order to do this we consider the path $\gamma: \mathbb{R} \rightarrow H_{2}^{\alpha}$ given by

$$
\gamma(t)=\left(\left(\alpha, Q_{1}(t)\right),\left(\alpha, Q_{2}\right),\left(\alpha, Q_{3}(t)\right),\left(\alpha, Q_{4}(t)\right)\right)
$$

where $Q_{1}(t)=\cos \left(\theta_{1}(t)\right) \mathbf{i}+\sin \left(\theta_{1}(t)\right) \mathbf{j}, Q_{2}=\mathbf{i}$ and $Q_{3}(t)=c(t) Q(t)+\cos \left(\theta_{2}(t)\right)$ $V_{1}(t)+\sin \left(\theta_{2}(t)\right) V_{2}(t)$. Here $c(t):=c\left(\alpha, \theta_{1}(t)\right), Q(t):=Q\left(\alpha, \theta_{1}(t)\right)$ and $V_{i}(t)$ are 
defined as in lemma 4.1. Note that $Q_{4}$ is determined by $Q_{1}, Q_{2}$ and $Q_{3}$. We choose $\theta_{1}(t)$ such that $\theta_{1}(0)=0$ and $\theta_{1}^{0}:=\left(d \theta_{1}(t) / d t\right)_{t=0} \neq 0$. This implies $\gamma(0)=\left(\mathbf{e}^{\mathbf{i} \alpha}, \mathbf{e}^{\mathbf{i} \alpha}, \mathbf{e}^{\mathbf{i} \alpha}, \mathbf{e}^{\mathbf{i} \alpha}\right)$ and $\gamma$ gives a smooth path $\widehat{\gamma}:(-\epsilon, 0) \rightarrow \widehat{H}_{2}^{\alpha}$ for a small $\epsilon>0$.

We call $\theta_{1}^{0}:=\theta_{1}^{0}(\widehat{\gamma}):=\left(d \theta_{1}(t) / d t\right)_{t=0}\left(\right.$ resp. $\left.\theta_{2}^{0}:=\theta_{2}^{0}(\widehat{\gamma}):=\theta_{2}(0)\right)$ the velocity (resp. angle) of $\widehat{\gamma}$ in $A$.

The derivative $\left(d Q_{3}(t) / d t\right)_{t=0} \cdot(-\mathbf{j}) \in \mathbb{C}$ can be written in the form $\theta_{1}^{0} \cdot s\left(\alpha, \theta_{2}^{0}\right)$ where

$$
\begin{aligned}
s\left(\alpha, \theta_{2}^{0}\right) & =z_{\alpha}+\cos \left(\theta_{2}^{0}\right)\left(1-z_{\alpha}\right)+\sin \left(\theta_{2}^{0}\right)\left(1-z_{\alpha}\right) \mathbf{i} \\
& =z_{\alpha}+\frac{1}{2 \cos \alpha} e^{\mathbf{i}\left(\theta_{2}^{0}+\alpha\right)} \\
& =\frac{\cos \left(\frac{2 \alpha+\theta_{2}^{0}}{2}\right)}{\cos \alpha} e^{\mathbf{i} \theta_{2}^{0} / 2} .
\end{aligned}
$$

Here $z_{\alpha}:=\frac{1}{2}-\frac{\mathbf{i}}{2} \tan \alpha \in \mathbb{C}$. By making use of the identification of $T_{\mathbf{e}}\left(\Sigma_{\alpha}\right)$ with $\mathbb{C}$ we obtain

$$
\gamma^{\prime}(t) \cdot(-\mathbf{j})=\theta_{1}^{0} \sin \alpha\left(1,0, s\left(\alpha, \theta_{2}^{0}\right), e^{-2 \mathbf{i} \alpha}\left(1-s\left(\alpha, \theta_{2}^{0}\right)\right) \in \mathbb{C}^{4} .\right.
$$

Note that $\theta_{2}^{0}(\widehat{\gamma})$ does not depend on the parameterization of $\widehat{\gamma}$.

Example 4.2. The angle of $\widehat{\Lambda}_{2}^{\alpha}$ in $A$ is 0 and the angle of $\widehat{\Gamma}_{\sigma_{1}^{-2}}^{\alpha}$ in $A$ is $-4 \alpha$. The latter can be seen as follows: by example 3.1 we have to look at

$$
\begin{aligned}
\frac{d}{d t}\left(\left(\alpha, Q_{2}\right)^{-1} Q_{1}(t)\left(\alpha, Q_{2}\right)\right)_{t=0} & =\frac{d}{d t}\left((\alpha, \mathbf{i})^{-1}(\cos t \mathbf{i}+\sin t \mathbf{j})(\alpha, \mathbf{i})\right)_{t=0} \\
& =(\alpha, \mathbf{i})^{-1}(\mathbf{j})(\alpha, \mathbf{i}) \\
& =e^{-2 \mathbf{i} \alpha} \mathbf{j} .
\end{aligned}
$$

This gives $\theta_{2}^{0}\left(\widehat{\Gamma}_{\sigma_{1}^{-2}}^{\alpha}\right)=-4 \alpha$ (see figure 3 ). Therefore, $\widehat{\Gamma}_{\sigma_{1}^{-2}}^{\alpha}$ is an arc in $\widehat{H}_{2}^{\alpha}$ connecting $A$ and $B^{\prime}$. It is easy to see that $\widehat{\Gamma}_{\sigma_{1}^{-2}}^{\alpha} \cap \widehat{\Lambda}_{2}^{\alpha}=\emptyset$ and since $\widehat{\Gamma}_{\sigma_{1}^{-2}}^{\alpha}$ is a graph, it intersects each circle $\theta_{2} \equiv$ constant in $\widehat{H}_{2}^{\alpha}$ in exactly one point (see figure 2). Moreover, $\widehat{\Gamma}_{\sigma_{1}^{-2}}^{\alpha}$ runs into $B^{\prime}$ as in the left hand side of figure 2 if $\pi / 2<\alpha<\pi$. The right hand side of figure 2 reflects the situation $0<\alpha<\pi / 2$. More precisely, let $\gamma:[0, \pi] \rightarrow \widehat{\Gamma}_{\sigma_{1}^{-2}}^{\alpha}$ be the parameterization of $\widehat{\Gamma}_{\sigma_{1}^{-2}}^{\alpha}, \gamma(t):=t e^{\mathbf{i} \theta_{2}(t)}$, where $\theta_{2}(0)=-4 \alpha$ and $\theta_{2}(\pi)=2 \pi$. A calculation as in equation (5) gives $\left(d \theta_{2}(t) / d t\right)_{t=\pi}=2 \epsilon(\alpha) \sin \alpha$ where $\epsilon(\alpha)$ is defined as in lemma 4.1 .

Let $K_{\alpha}$ be the circle in $\mathbb{C}$ with center $z_{\alpha}$ and radius $1 /(2 \cos \alpha)$. Then $s\left(\alpha, \theta_{2}^{0}\right) \in$ $K_{\alpha}$ in particular we have $0,1, e^{-2 \mathbf{i} \alpha} \in K_{\alpha}$. 

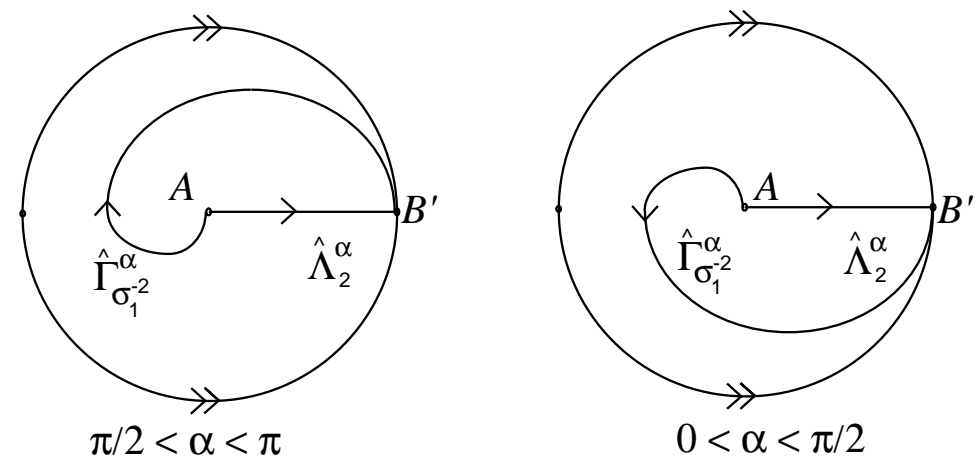

Figure 2 .

The difference cycle $\left(\widehat{\Gamma}_{\sigma_{1}^{-2}}^{\alpha}-\widehat{\Lambda}_{2}^{\alpha}\right)$.

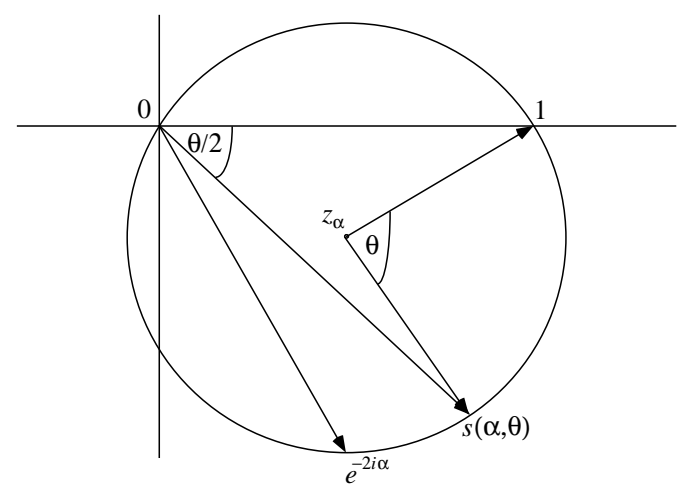

Figure 3.

The circle $K_{\alpha} \subset \mathbb{C}$.

Equation (5) tells us that for a given $s \in K_{\alpha}$ the $\theta_{2}$-parameter of $s$ is determined by the $\operatorname{argument} \arg s$ where $0 \leq \arg s<2 \pi$. More precisely we have $\theta_{2}=2 \arg s$ (see figure 3 ). Note that $\theta_{2}(0) \equiv \pi-2 \alpha \bmod 2 \pi$ is well defined.

Later we will make use of the following observation:

Lemma 4.3. Let $s \in K_{\alpha}$ be given. Then $f_{\alpha}(s):=\frac{e^{2 \alpha_{s}-1}}{s-1}$ is a real number.

More precisely, $f_{\alpha}^{-1}\left(\mathbb{R}_{<0}\right) \subset K_{\alpha}$ is the open arc between 1 and $e^{-2 \mathbf{i} \alpha}$ which does not contain the 0 . 
Proof. The Moebius transformation

$$
f_{\alpha}: z \mapsto \frac{e^{2 \mathbf{i} \alpha} z-1}{z-1}
$$

maps $K_{\alpha}$ into the real line. The conclusion of the lemma follows because $f_{\alpha}(0)=1$, $f_{\alpha}\left(e^{-2 \mathbf{i} \alpha}\right)=0$ and $f_{\alpha}(1)=\infty$ (see figure 3$)$.

Let $\mathbf{a}:=\left(\mathbf{e}^{2 \mathbf{i} \alpha}, \ldots, \mathbf{e}^{2 \mathbf{i} \alpha}\right) \in S_{n}^{\alpha}$ be given. The point $\mathbf{a}$ is fixed by $\sigma: R_{n}^{\alpha} \rightarrow R_{n}^{\alpha}$. The derivative of $\sigma$ at $\mathbf{a}$ is given by the Burau-matrix. More precisely we have the following commutative diagram (see [Lon89] and [Lin92])

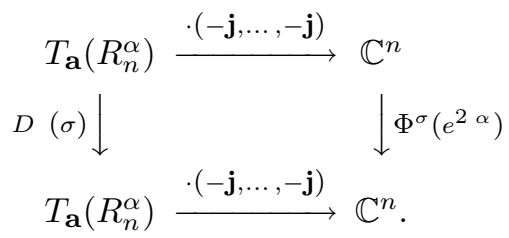

We write $\Phi^{\sigma}\left(e^{2 \mathbf{i} \alpha}\right)$ in the form

$$
\Phi^{\sigma}\left(e^{2 \mathbf{i} \alpha}\right)=\left(\begin{array}{ll}
\mathbf{A} & \mathbf{B} \\
\mathbf{C} & \mathbf{D}
\end{array}\right)
$$

where $\mathbf{D}\left(e^{2 \mathbf{i} \alpha}\right)$ is a $(n-2) \times(n-2)$ matrix.

Lemma 4.4. Let $\sigma \in \mathfrak{B}_{n}$ be given such that $k=\sigma^{\wedge}$ is a knot. Moreover, assume that $\alpha \in(0, \pi)$ is given such that $e^{2 \mathbf{i} n \alpha} \neq 1$.

If $\Delta_{k}\left(e^{2 \mathbf{i} \alpha}\right) \neq 0$ then $\operatorname{det}\left(\mathbf{D}\left(e^{2 \mathbf{i} \alpha}\right)-\mathbf{E}\right) \neq 0$.

Proof. The normalized Alexander polynomial of $k:=\sigma^{\wedge}$ is given by

$$
\Delta_{k}(t)=\left(-\frac{1}{\sqrt{t}}\right)^{e-n+1} \frac{1-t}{1-t^{n}} \operatorname{det}\left(\mathbf{E}-\phi^{\sigma}(t)\right)
$$

where $e$ is the exponent sum of $\sigma$ (see [Jon87]). Note that $\Delta_{k}(t)=\Delta_{k}\left(t^{-1}\right)$ and $\Delta_{k}(1)=1$. From equation (6) and lemma 2.2 the following correspondence can be derived

$$
\Delta_{k}(t)=(-1)^{e}\left(\frac{1}{\sqrt{t}}\right)^{e-n+1} c_{1,1}^{\sigma}(t)
$$

Assume $\operatorname{det}\left(\mathbf{D}\left(e^{2 \mathbf{i} \alpha}\right)-\mathbf{E}\right)=0$ then by lemma 2.2 we have $c_{1,1}^{\sigma_{1}^{2} \sigma}\left(e^{2 \mathbf{i} \alpha}\right)=$ $e^{4 \mathbf{i} \alpha} c_{1,1}^{\sigma}\left(e^{2 \mathbf{i} \alpha}\right)$.

From this we deduce the formula

$$
\Delta_{k^{\prime}}\left(e^{2 \mathbf{i} \alpha}\right)=e^{2 \mathbf{i} \alpha} \cdot \Delta_{k}\left(e^{2 \mathbf{i} \alpha}\right) .
$$


This gives a contradiction if $e^{2 \mathbf{i} \alpha} \neq-1$ because $\Delta_{k}\left(e^{2 \mathbf{i} \alpha}\right), \Delta_{k^{\prime}}\left(e^{2 \mathbf{i} \alpha}\right) \in \mathbb{R}$ and $e^{2 \mathbf{i} \alpha} \notin \mathbb{R}$.

If $e^{2 \mathbf{i} \alpha}=-1$ then we have $\Delta_{k}(-1)=-\Delta_{k^{\prime}}(-1)$. Now $k$ and $k^{\prime}$ differ only by a crossing change and therefore

$$
\Delta_{k}(-1)-\Delta_{k^{\prime}}(-1)=2 \mathbf{i} \Delta_{k^{\prime \prime}}(-1) \Longrightarrow \Delta_{k}(-1)=\mathbf{i} \Delta_{k^{\prime \prime}}(-1)
$$

where $k^{\prime \prime}:=\left(\sigma_{1} \sigma\right)^{\wedge}$ is a 2-component link. Let $\widetilde{\Delta}_{k^{\prime \prime}}(t)$ be the Hosokawa polynomial of $k^{\prime \prime}$. The connection with the Alexander polynomial is given by

$$
\Delta_{k^{\prime \prime}}(t)=\left(\sqrt{t}-\frac{1}{\sqrt{t}}\right) \widetilde{\Delta}_{k^{\prime \prime}}(t)
$$

(see [Mur96]). Since the Hosokawa polynomial is a symmetric integer polynomial we obtain from (8) and (9):

$$
\Delta_{k}(-1)=\mathbf{i} \Delta_{k^{\prime \prime}}(-1)=-2 \widetilde{\Delta}_{k^{\prime \prime}}(-1)
$$

Therefore we have $\Delta_{k}(-1) \equiv 0 \bmod 2$ which is impossible.

As in the trace-free case we investigate the projection $\widehat{p}\left(\widehat{\Gamma}_{\sigma}^{\alpha}\right)$ in a neighborhood of $A$ (see [Lin92]).

Lemma 4.5. Choose $n \in \mathbb{N}$ such that $e^{2 \mathrm{i} n \alpha} \neq 1$. Then in a neighborhood of $A$ on $\widehat{H}_{2}^{\alpha}, \widehat{p}\left(\widehat{\Gamma}_{\sigma}^{\alpha} \cap \widehat{V}_{n}^{\alpha}\right)$ is a curve approaching $A$.

Moreover, the angle $\theta_{2}^{0}$ of $\widehat{p}\left(\widehat{\Gamma}_{\sigma}^{\alpha} \cap \widehat{V}_{n}^{\alpha}\right)$ at $A$ is not equal to 0 or $-4 \alpha$.

Proof. The main steps of the proof are analogous to Lin's proof (see [Lin92, Lemma 2.4]). But by lemma 4.4 we have only to consider Case 1 which simplifies the trails of the proof.

Let $\theta_{2}^{0}$ be the angle of $\widehat{p}\left(\widehat{\Gamma}_{\sigma}^{\alpha} \cap \widehat{V}_{n}^{\alpha}\right)$ at $A$. We have $\theta_{2}^{0}=2 \arg s_{\sigma}$ where the parameter $s_{\sigma}$ is given by

$$
\Phi^{\sigma}\left(e^{2 \mathbf{i} \alpha}\right)\left(\begin{array}{c}
1 \\
0 \\
\mathbf{v}
\end{array}\right)=\left(\begin{array}{c}
s_{\sigma} \\
e^{-2 \mathbf{i} \alpha}\left(1-s_{\sigma}\right) \\
\mathbf{v}
\end{array}\right) .
$$

Here

$$
\mathbf{v}=(\mathbf{E}-\mathbf{D})^{-1} \mathbf{C}\left(\begin{array}{l}
1 \\
0
\end{array}\right)
$$

Since we have chosen $e^{2 \mathbf{i} \alpha}$ such that $\Delta_{k}\left(e^{2 \mathbf{i} \alpha}\right) \neq 0 \neq \Delta_{k^{\prime}}\left(e^{2 \mathbf{i} \alpha}\right)$ the second statement of the lemma 4.5 follows from the same conclusions as Lin's proof. 
Lemma 4.6. $h^{\alpha}\left(\sigma_{1}^{2} \sigma\right)-h^{\alpha}(\sigma)=\epsilon$, where

$$
\epsilon= \begin{cases}0 & \text { if } s_{\sigma} \in f_{\alpha}^{-1}\left(\mathbb{R}_{>0}\right) \\ 1 & \text { if } s_{\sigma} \in f_{\alpha}^{-1}\left(\mathbb{R}_{<0}\right)\end{cases}
$$

Proof. We start by a perturbation $\widehat{\Gamma}_{\sigma}^{\alpha} \rightsquigarrow \breve{\Gamma}_{\sigma}^{\alpha}$ with compact support such that $\breve{\Gamma}_{\sigma}^{\alpha} \pitchfork\left(\widehat{\Gamma}_{\sigma_{1}^{-2}}^{\alpha}-\widehat{\Lambda}_{n}^{\alpha}\right)$. We claim that there is a neighborhood of $B^{\prime}=(\pi, 0)$ on $\widehat{H}_{2}^{\alpha}$ such that $\widehat{p}\left(\breve{\Gamma}_{\sigma}^{\alpha} \cap \widehat{V}_{n}^{\alpha}\right)$ is disjoint with that neighborhood. Suppose this is not true. Then we will get a point in $\breve{\Gamma}_{\sigma}^{\alpha} \cap \widehat{V}_{n}^{\alpha}$ which is represented by $(\mathbf{A}, \mathbf{A})$ where $\mathbf{A}:=\left(\mathbf{e}^{2 \mathbf{i} \alpha}, \mathbf{e}^{-2 \mathbf{i} \alpha}, A_{3}, \ldots, A_{n}\right)$. But $(\mathbf{A}, \mathbf{A})$ represents also a point of $\widehat{W}_{n}^{\alpha}$. This gives a contradiction if $(\mathbf{A}, \mathbf{A})$ represents an irreducible point (remember: $\left.\widehat{W}_{n}^{\alpha} \cap \breve{\Gamma}_{\sigma}^{\alpha}=\emptyset\right)$. If $(\mathbf{A}, \mathbf{A})$ represents an reducible point then this point is already in $\Gamma_{\sigma}^{\alpha} \cap V_{n}^{\alpha}$ since the isotopy above has compact support. Therefore, $\mathbf{A}$ would be a fixed point of $\left.\sigma\right|_{R_{n}^{\alpha}}$. But this is impossible since the permutation induced by $\sigma$ is a $n$-cycle.

We continue by a small perturbation relative to a neighborhood of the reducible point $\left(\mathbf{e}^{\mathbf{i} \alpha}, \ldots, \mathbf{e}^{\mathbf{i} \alpha}\right)$ changing $\breve{\Gamma}_{\sigma}^{\alpha}$ to $\widetilde{\Gamma}_{\sigma}^{\alpha}$ such that $\widetilde{\Gamma}_{\sigma}^{\alpha} \pitchfork \widehat{V}_{n}^{\alpha}$. Then $\widehat{p}\left(\widetilde{\Gamma}_{\sigma}^{\alpha} \cap \widehat{V}_{n}^{\alpha}\right)$ is a 1dimensional submanifold of $\widehat{H}_{2}^{\alpha}$. In a neighborhood of $A$, it is a curve approaching $A$ with angle $\theta_{2}^{0}=2 \arg s_{\sigma}$. The other end of the curve must approach $B$. Since

$$
h^{\alpha}\left(\sigma_{1}^{2} \sigma\right)-h^{\alpha}(\sigma)=\left\langle\widehat{\Gamma}_{\sigma_{1}^{-2}}^{\alpha}-\widehat{\Lambda}_{2}, \widehat{p}\left(\widetilde{\Gamma}_{\sigma}^{\alpha}\right)\right\rangle_{\widehat{H}_{2}^{\alpha}}
$$

and the angle of $\widehat{\Lambda}_{\sigma}^{\alpha}\left(\right.$ resp. $\left.\widehat{\Gamma}_{\sigma_{1}^{-2}}^{\alpha}\right)$ at $A$ is $0($ resp. $-4 \alpha)$ the conclusion of the lemma follows (see figure 2).

Lemma 4.7. Under the assumptions of lemma 4.5 we have

$$
\frac{\Delta_{k^{\prime}}\left(e^{2 \mathbf{i} \alpha}\right)}{\Delta_{k}\left(e^{2 \mathbf{i} \alpha}\right)}=\frac{e^{2 \mathbf{i} \alpha} s_{\sigma}-1}{s_{\sigma}-1}=f_{\alpha}\left(s_{\sigma}\right) .
$$

Proof. The proof is completely analogous to Lin's proof and again the fact $\operatorname{det}(\mathbf{D}-$ E) $\neq 0$ simplifies the argument.

We are now able to state the main result of this section

Proposition 4.8. Let $k_{-}=\left(\sigma_{1}^{2} \sigma\right)^{\wedge}$ and $k_{+}=(\sigma)^{\wedge}$ be knots. Moreover, let $\alpha \in(0, \pi)$ be given such that $\Delta_{k_{+}}\left(e^{2 \mathbf{i} \alpha}\right) \neq 0 \neq \Delta_{k_{-}}\left(e^{2 \mathbf{i} \alpha}\right)$. Then

$$
h^{\alpha}\left(k_{-}\right)-h^{\alpha}\left(k_{+}\right)= \begin{cases}0 & \text { iff } \Delta_{k_{+}}\left(e^{2 \mathbf{i} \alpha}\right) \cdot \Delta_{k_{-}}\left(e^{2 \mathbf{i} \alpha}\right)>0 \\ 1 & \text { iff } \Delta_{k_{+}}\left(e^{2 \mathbf{i} \alpha}\right) \cdot \Delta_{k_{-}}\left(e^{2 \mathbf{i} \alpha}\right)<0\end{cases}
$$


Proof. Assume again that $e^{2 \mathbf{i} n \alpha} \neq 1$.

Lemma 4.7 gives us

$$
f_{\alpha}\left(s_{\sigma}\right)>0 \text { iff } \Delta_{k_{-}}\left(e^{2 \mathbf{i} \alpha}\right) \cdot \Delta_{k_{+}}\left(e^{2 \mathbf{i} \alpha}\right)>0
$$

and

$$
f_{\alpha}\left(s_{\sigma}\right)<0 \text { iff } \Delta_{k_{-}}\left(e^{2 \mathbf{i} \alpha}\right) \cdot \Delta_{k_{+}}\left(e^{2 \mathbf{i} \alpha}\right)<0
$$

where $f_{\alpha}$ is the Moebius transformation defined in lemma 4.3. The result follows now from lemma 4.6.

There is also a recursive procedure for calculating the value of the signature function. Let $\omega \in S^{1}$ such that $\Delta_{k}(\omega) \neq 0$. The signature $\sigma_{k}(\omega)$ is always an even integer. We have

$$
\sigma_{k}(\omega) \equiv 0 \bmod 4 \text { iff } \Delta_{k}(\omega)>0 \quad \text { and } \quad \sigma_{k}(\omega) \equiv 2 \bmod 4 \text { iff } \Delta_{k}(\omega)<0 .
$$

Moreover, for knots $k_{+}$and $k_{-}$we obtain from simple consideration of the Seifert matrices of $k_{+}$and $k_{-}$

$$
0 \leq \sigma_{k_{-}}(\omega)-\sigma_{k_{+}}(\omega) \leq 2
$$

(see [Lip90] and [Gil82]).

We are ready to proof the main result of this section:

Proof of theorem 1.2. The invariants $h^{\alpha}(k)$ and $\sigma_{k}\left(e^{2 \mathbf{i} \alpha}\right)$ are defined and they are both locally constant. Choose an irrational angle $\beta \in(0, \pi), \beta<\alpha$ such that $\Delta_{k}\left(e^{2 \mathbf{i} t}\right) \neq 0$ for all $t \in[\beta, \alpha]$.

It follows that $h^{\alpha}(k)=h^{\beta}(k)$ and $\sigma_{k}\left(e^{2 \mathbf{i} \alpha}\right)=\sigma_{k}\left(e^{2 \mathbf{i} \beta}\right)$. Since $\beta$ is irrational we have $\Delta_{k^{\prime}}\left(e^{2 \mathbf{i} \beta}\right) \neq 0$ for every knot $k^{\prime} \subset S^{3}$.

The inductive procedure given by proposition 4.8 and by equations (10) and (11) makes it possible to prove that

$$
h^{\beta}(k)=\frac{1}{2} \sigma_{k}\left(e^{2 \mathbf{i} \beta}\right) .
$$

\section{Remark 4.9.}

1. Note that theorem 1.2 includes the case $\alpha=\pi / 2$ because $\Delta_{k}(-1) \neq 0$.

2. We are not able to calculate the value of $h^{\alpha}(k)$ if $\widehat{\Lambda}_{n}^{\alpha} \cap \widehat{\Gamma}_{\sigma}^{\alpha}$ is compact but $\Delta_{k}\left(e^{2 \mathbf{i} \alpha}\right)=0$.

\section{Acknowledgments}

The authors are grateful to Uwe Kaiser for carefully reading the manuscript and suggesting several improvements. The first author wishes to express his gratitude to Hans Boden, Chris Herald, Paul Kirk, Eric Klassen, Chuck Livingston and Klaus Mohnke for helpful discussions on subjects related to this paper. 


\section{References}

[Bur90] G. Burde, SU(2)-representation spaces for two-bridge knot groups, Math. Ann. 288 (1990), 103-119.

[BZ85] G. Burde and H. Zieschang, Knots, Walter de Gruyter, 1985.

[FK91] C. D. Frohman and E. P. Klassen, Deforming representations of knot groups in SU(2), Comment. Math. Helvetici 66 (1991).

[Gil82] C. Giller, A family of links and the Conway calculus, Trans. AMS 270 (1982), 75-109.

[GM92] L. Guillou and A. Marin, Notes sur l'invariant de Casson des sphères d'homologie de dimension trois, L'Enseignement Math. 38 (1992), 233-290.

[Gor78] C. Mc A. Gordon, Some aspects of classical knot theory. In: Knot Theory, Lecture Notes in Mathematics 685, 1978.

[Her97] Ch. M. Herald, Existence of irreducible representations for homology knot complements with nonconstant equivariant signature, Math. Ann. 309 (1997).

[Heu97] M. Heusener, An orientation for the SU(2)-representation space of knot groups, Preprint, 1997.

[Jon87] V. F. R. Jones, Hecke algebra representations of braid groups and link polynomials, Ann. of Math. 126 (1987), 355-388.

[Kau87] L. H. Kauffman, On Knots, Annals of Mathematics Studies 115, Princeton University Press, 1987.

[Kla91] E. P. Klassen, Representations of knot groups in SU(2), Transactions of the AMS 326(2) (1991).

[Kro96] J. Kroll, Äquivariante Signatur und SU(2)-Darstellungsräume von Knotengruppen, Diplomarbeit, Universität-Gesamthochschule Siegen, 1996.

[Lin92] Xiao-Song Lin, A knot invariant via representation spaces, J. Differential Geometry 35 (1992).

[Lip90] A. S. Lipson, Link signature, Goeritz matrices and polynomial invariants, L'Enseignement Math. 36 (1990), 93-114.

[Lon89] D. D. Long, On the linear representation of braid groups, Transactions of the AMS 311 (1989), 535-560.

[Mur96] K. Murasugi, Knot Theory and its Applications, Birkhäuser, 1996.

Michael Heusener

Université Paul Sabatier

Laboratoire Emile Picard

118, route de Narbonne

F-31062 Toulouse-Cedex

France

e-mail: heusener@picard.ups-tlse.fr
Jochen Kroll

Uni-GH-Siegen

Fachbereich Mathematik

Hölderlinstraße 3

D-57068 Siegen

Germany

e-mail: kroll@mathematik.uni-siegen.de

(Received: December 1, 1997) 